\title{
Os caminhos da reformulação do Projeto Pedagógico da Faculdade de Odontologia da Universidade Federal do Amazonas
}

\author{
Emílio Carlos Sponchiado Júnior*; Nikeila Chacon de Oliveira Conde*; Izabelly Esteves Bittencourt \\ Martins**; Flávia Cohen Carneiro*; Janete Maria Rebelo Vieira*; Maria Augusta Bessa Rebelo***
}

* Professor Associado, Curso de Odontologia, Universidade Federal do Amazonas

** Mestranda, Programa de Pós-Graduação em Odontologia, Universidade Federal do Amazonas

*** Professora Titular, Curso de Odontologia, Universidade Federal do Amazonas

Recebido em 15/05/2018. Aprovado em 01/06/2019.

\begin{abstract}
RESUMO
Este artigo relata a experiência de uma Faculdade de Odontologia durante o processo de construção e implementação de um novo projeto pedagógico (PP). O PP foi reformulado desde a caracterização do curso com as discussões sobre perfil de egresso, passando pela estrutura de funcionamento, objetivos, matriz curricular, estágios e concepção metodológica. Durante 12 meses foram utilizadas diversas ferramentas para sensibilização do corpo docente, incluindo a realização de semanas pedagógicas, oficinas, encontros pedagógicos e discussões nas assembleias docentes. $\mathrm{O}$ novo currículo foi implementado em 2012 evidenciando a interdisciplinaridade, a formatação de ambulatórios integrados e um estágio curricular obrigatório que compreende mais de $40 \%$ da matriz curricular entre atividades intra e extramuros. Dificuldades foram encontradas durante a implementação e a comunidade acadêmica buscou ferramentas para minimizá-las, fazendo com que o projeto continue dinâmico e amadureça a cada ano.
\end{abstract}

Descritores: Educação. Odontologia. Currículo.

\section{INTRODUÇÃO}

Este artigo relata a vivência dos atores da Faculdade de Odontologia da Universidade
Federal Amazonas (UFAM) durante a construção do atual projeto político pedagógico do Curso de Odontologia. 


\section{RELATO DE EXPERIÊNCIA}

\section{O Contexto favorável para mudanças}

O curso de Odontologia da UFAM foi criado em 1966. Funcionava no prédio da Escola Estadual Nilo Peçanha e, posteriormente, foi transferido para o Bairro de Aparecida, junto com o curso de Farmácia. No ano de 1980 houve a criação da Faculdade de Ciências da Saúde, composta pelos cursos de Odontologia, Farmácia e Medicina. Desde então, a Odontologia foi transferida para o bairro Praça XIV de Janeiro, onde funciona até os dias atuais ${ }^{1}$.

Em 25 de julho de 2007, foi criada a Faculdade de Odontologia, no ato do desmembramento da Faculdade de Ciências da Saúde a qual pertenciam os cursos de Farmácia, Odontologia e Medicina. A nova unidade que abrigava somente o curso de Odontologia optou pelo sistema de organização não departamental.

O curso de Odontologia possuía em sua estrutura curricular disciplinas do ciclo básico que eram ministradas por outras unidades acadêmicas, ainda estruturadas pelo sistema departamental, como o Instituto de Ciências Biológicas, Instituto de Ciências Exatas, Instituto de Ciências Humanas e Letras, dentre outros, e este fato trazia dificuldades para a integração dos conteúdos.

A partir deste marco, o corpo docente se preparou para a elaboração de um novo projeto pedagógico de curso (PPC) que contemplasse efetivamente as Diretrizes Curriculares Nacionais (DCN), considerando que havia um movimento nacional para que os Cursos de Odontologia pudessem cumprir estas diretrizes ${ }^{2}$.

A primeira ação da administração foi criar o Núcleo Docente Estruturante (NDE) para iniciar os trabalhos de reformulação do PPC. Motivados pela mudança administrativa da Faculdade com uma hierarquia organizacional bem definida o NDE avançou com seu planejamento e estratégia para a reformulação.
Para tanto, foram resgatados históricos e experiências de encontros ocorridos anteriormente na Unidade por meio de oficinas com consultores externos vinculados à Associação Brasileira de Ensino Odontológico - ABENO, entre os anos de 2003 e 2007, onde se discutiu as fragilidades do currículo, os nós críticos e as medidas necessárias para a adequação às novas diretrizes.

Em 2008, diante das observações durante o processo de ensino-aprendizagem e do acompanhamento das atividades docentes no âmbito das competências da Coordenação Acadêmica, Coordenação de Curso e NDE, idealizou-se uma Semana Pedagógica.

Com a temática central "A Docência no Ensino Superior" o encontro teve como objetivos principais a integração profissional e relacionamento interpessoal, discussão dos métodos de ensino e avaliação, a valorização da docência e criação de protocolos de conduta clínica de temas divergentes entre os docentes, visando preparar o corpo docente para no futuro ser realizada a reconstrução do PPC.

Em 2009 e 2010 foram realizadas outras Semanas Pedagógicas, cujo tema central foi a Reformulação Curricular, com a participação de $100 \%$ dos docentes da Unidade, representantes de Disciplinas do Ciclo Básico, Pedagogas, representantes acadêmicos de cada período e egressos, ou seja, com adesão de todos os atores para a construção do projeto, fato raro em situações semelhantes ${ }^{3}$.

Durante esses encontros baseados em todo o processo vivenciado, foi iniciada a construção coletiva da nova proposta de currículo integrado reformulando as caracterizações do curso (perfil do egresso, competências e habilidades profissionais e objetivos do curso) a matriz curricular, a concepção metodológica (métodos de avaliação do ensino e do projeto pedagógico), além da infraestrutura e do corpo docente e técnico administrativo.

$\mathrm{Na}$ última oficina, em 2010, a faculdade 
recebeu consultores da ABENO, para avaliação e discussão da proposta do novo projeto pedagógico. A participação de todos foi essencial e primordial para a concretização deste objetivo, onde foi formulada uma minuta de propostas, posteriormente trabalhada pelo NDE.

A etapa que dispendeu mais tempo e reflexão foi a de estabelecer um calendário de encontros para identificar os eixos temáticos, construir a estrutura curricular, ementas, objetivos e bibliografias para cada nova disciplina do currículo.

Desta forma, os encontros ocorreram sistematicamente aos sábados ou em período noturno, com a participação de professores efetivos do curso e membros do NDE. Estas reuniões foram extremamente importantes, pois foi o primeiro momento em que docentes de diferentes disciplinas se reuniram para montar uma ementa única para disciplinas antes isoladas.

Em todas as fases da construção os professores de todas as Unidades envolvidas foram convidados. Ao longo do processo, mudanças mais significativas em disciplinas do Ciclo Básico não foram possíveis por razões administrativas, já que eram ofertadas e coordenadas por outras unidades ainda no sistema departamental.

O novo projeto pedagógico foi aprovado pela comunidade da faculdade e pelo Conselho Superior de Ensino, Pesquisa e Extensão em 2011, sendo implementado no primeiro semestre de 2012, como currículo único do Curso de Odontologia.

\section{Dados gerais do projeto}

As discussões a respeito de mudanças no PPC ocorriam há anos no Curso de Odontologia. Ao longo desse tempo foram estabelecidos diagnósticos a respeito das principais deficiências do antigo projeto pedagógico, baseado no paradigma cartesiano, dentre os quais destacam-se: grande concentração de conteúdos de caráter técnico e pouca ênfase na área de ciências humanas e sociais; técnicas pedagógicas centradas na figura do professor; inexistência de articulação entre as diversas áreas ou disciplinas; modelo de clínica odontológica fragmentada; ausência de mecanismos que facilitassem a participação do aluno em atividades do curso; concepções conservadoras de avaliação com modelos baseados na memorização ${ }^{3}$.

Estas deficiências eram resultado do modelo de formação nos diversos cursos de odontologia do país com tendência para uma formação elitista, voltada para a especialização e sem nenhum interesse para com o serviço público ${ }^{4}$.

Buscando atender aos conteúdos essenciais para o curso de Odontologia que devem estar relacionados com o processo saúde-doença do ser humano, conforme preconizam as diretrizes curriculares nacionais, a nova matriz curricular teve como eixo estruturador a integração entre "saúde e sociedade".

Um dos pilares do ensino é a área de promoção da saúde, decorrente do conhecimento de que doença não é só uma questão biológica, mas um processo sócio-histórico-ambiental que se manifesta no biológico.

Por este motivo a área de Saúde Coletiva está presente ao longo do curso, com crescente complexidade do conteúdo programático proposto, destacando o estudo de temas, questões e problemas próprios da saúde coletiva e do sistema público de saúde.

A realidade da prática no Sistema Único de Saúde foi o ponto de partida para o desenvolvimento das atividades curriculares, do início até o final do Curso. Durantes os momentos de diálogo e construção do novo projeto foi enfatizado tais práticas para que as mudanças não se limitassem somente na alteração de carga horaria ou ao simples reposicionamento de disciplinas, todas as ferramentas possíveis foram utilizadas para que se aprofundasse a discussão sobre a responsabilidade da formação do perfil do egresso 
conforme as novas diretrizes, situação esta também vivenciada e relatada pelo curso de Odontologia da USS, em Vassouras/RJ na década passada ${ }^{5}$.

O perfil do egresso formado pelo novo PPC foi de um profissional, com formação generalista, humanista, crítica e reflexiva, para atuar em todos os níveis de atenção à saúde, com base no rigor técnico e científico. A partir deste contexto a matriz curricular foi delineada.

\section{Matriz Curricular: Eixos estruturantes do Currículo}

Os conteúdos essenciais do Curso de Graduação em Odontologia foram relacionados com todo o processo saúde-doença do cidadão, da família e da comunidade, integrado à realidade epidemiológica e profissional da região. Os conteúdos foram alicerçados em quatro eixos principais (figura 1).

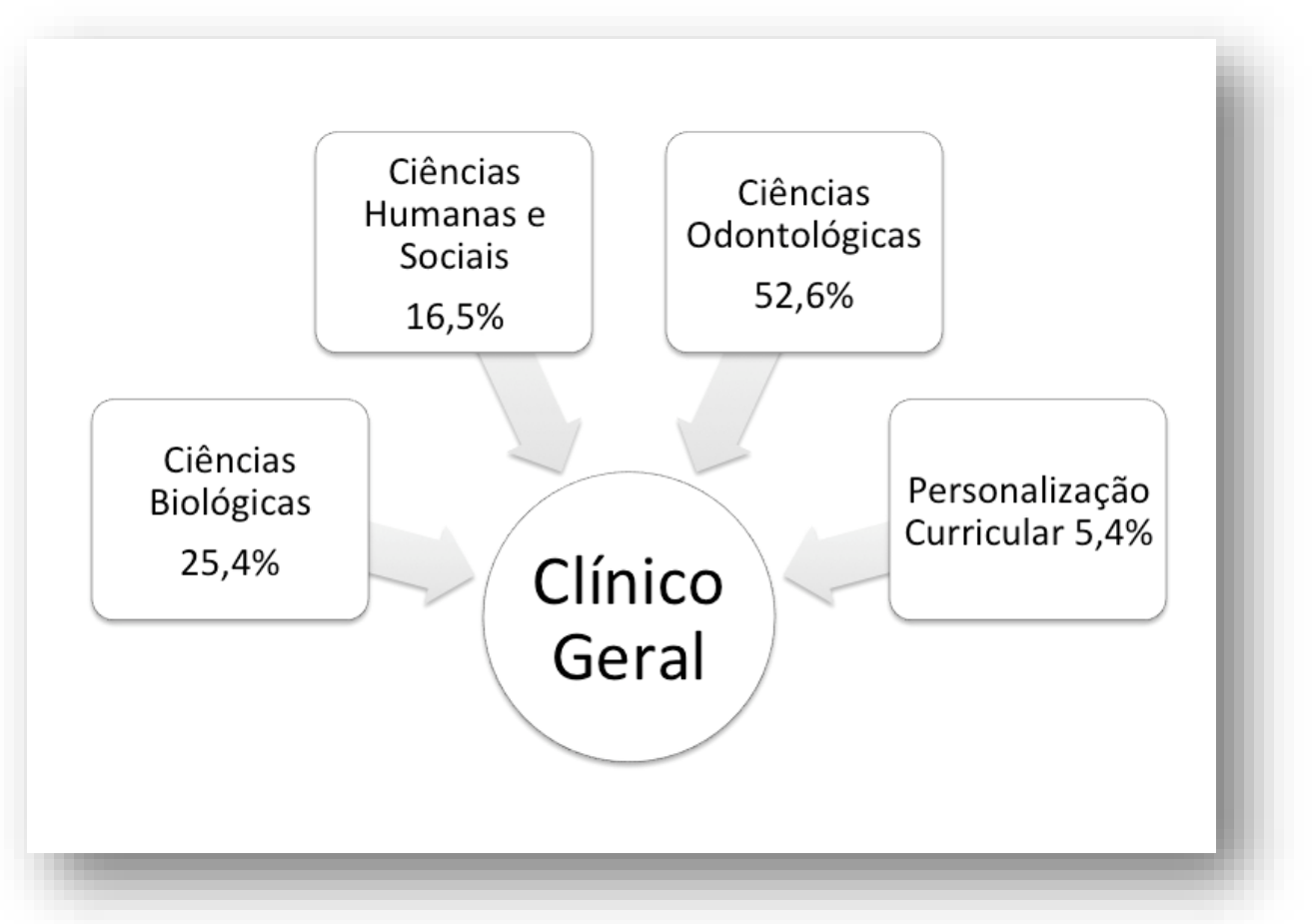

Figura 1. Eixos estruturantes do currículo

$\mathrm{O}$ eixo de Ciências Biológicas congrega disciplinas com conteúdos de bases moleculares e celulares dos processos normais e alterados, da estrutura e função dos tecidos, órgãos, sistemas e aparelhos, aplicados às situações decorrentes do processo saúde-doença no desenvolvimento da prática assistencial da Odontologia.

O eixo de Ciências Humanas e Sociais aglutina conteúdos referentes às diversas dimensões da relação indivíduo/sociedade, contribuindo para a compreensão dos determinantes sociais, culturais, comportamentais, psicológicos, ecológicos, éticos e legais, nos níveis individual e coletivo, do processo saúde-doença; bem como o gerenciamento da carreira profissional do egresso.

O eixo de Ciências Odontológicas contempla os conteúdos de Propedêutica Clínica, Clínica Odontológica e Odontologia Pediátrica. 
O Eixo de Personalização Curricular compreende conteúdos de execução do Trabalho de Conclusão do Curso, das Disciplinas Optativas e das Atividades Acadêmicas Complementares.

\section{O planejamento para execução do Estágio Curricular}

O estágio curricular foi o instrumento de integração e conhecimento do discente à realidade social e econômica da região. No contexto deste projeto foi entendido como a assistência integral ao paciente prestada a usuários da comunidade pelo estudante de Odontologia, em atividades intra e extramuros. Também pode ser cumprido em atendimentos multidisciplinares e em serviços assistenciais públicos e privados. Seu objetivo foi fomentar a relação ensino-serviços, ampliar as relações da universidade com a sociedade e propiciar ao futuro profissional contato com as diversas realidades sociais, consolidando o espaço pedagógico ${ }^{6,7}$.

A partir desse conceito, o Estágio Curricular foi planejado para ter 1770 horas (300h teóricas; $1170 \mathrm{~h}$ de prática intramuros; e $300 \mathrm{~h}$ de prática extramuros), perfazendo $43,75 \%$ da carga horária total do curso, distribuído ao longo dos 7 últimos semestres letivos.

Nos dois primeiros semestres e no quinto semestre letivo são trabalhados conhecimentos teóricos e práticos da área de Saúde Coletiva (o sistema de saúde brasileiro, o entendimento sobre o processo saúde-doença e epidemiologia), de forma a preparar o estudante para as atividades de prática extramuros $^{8-10}$.

No quarto semestre são iniciadas as atividades intramuros., com a oferta de disciplinas como Estomatologia Clínica, e posteriormente, Clínicas Integradas I, II, III e IV e Clínicas Odontológicas Infantis I e II. A partir do quinto semestre começam as atividades extramuros com as disciplinas Saúde Bucal Coletiva V e VI.

$\mathrm{Na}$ primeira os estudantes participam por meio de observação nos serviços públicos de saúde, tanto municipal quanto estadual, visitando unidades e participando de ações educativas.

Na disciplina de Saúde Bucal Coletiva VI, as atividades são divididas em módulos. Os discentes são apresentados a diferentes realidades da zona rural do Amazonas, bem como desenvolvem atividades nas unidades de saúde da Secretaria Municipal de Saúde de Manaus e em Centros de Referência como a Fundação Hospitalar de Hematologia e Hemoterapia do Amazonas (HEMOAM) e Hospitais Universitários.

A importância da aproximação dos discentes, durante sua formação, com a comunidade local em que atuará profissionalmente é apontada no estudo de Salvador; Sant'Ana (2017) ${ }^{11}$ como uma necessidade para que os futuros profissionais consigam cumprir o objetivo de manter a assistência integral à saúde da população, conforme requer um projeto pedagógico permeado por experiências em que os atores vivenciem esta problemática.

\section{Concepção Metodológica}

Com a reformulação do projeto pedagógico era esperado que o docente pudesse ser capacitado a exercer sua atividade não mais com o método da educação "bancária", na qual o aluno é tratado como um depósito de conhecimentos, sendo passivo no recebimento das informações, não conseguindo observar, analisar e questionar a sua realidade ${ }^{4,5}$.

Assim, no atual PPC, foi resgatado um modelo de formação com uso de metodologias pedagógicas inovadoras e centradas no estudante, de forma articulada com a ciência e os problemas sociais e ainda como possibilidade de troca do método da pedagogia de transmissão pelo método da pedagogia da problematização ou outras metodologias ativas ${ }^{12}$.

Foram realizados por dois anos consecutivos (2012-2013) treinamentos com os docentes visando à aplicação de metodologias ativas em suas 
disciplinas. Após esse período o Colegiado do Curso começou acompanhar a incorporação destas metodologias ativas a cada semestre em todas disciplinas do currículo.

A sistemática de funcionamento das Clínicas Integradas seguiu o princípio da complexidade crescente a cada período, sempre tendo como suporte teórico as disciplinas oferecidas no período anterior e com caráter cumulativo, porém para o funcionamento pleno das clínicas a unidade necessitava de um setor de triagem informatizado. Esta etapa sofreu atrasos de implementação, por dificuldades com recursos humanos para se operacionalizar o setor. O ensino em Clínicas Integradas contempla a metodologia baseada em problemas, muito utilizada em escolas de Medicina que buscam uma formação diferenciada para seus alunos $^{13}$.

O novo currículo entrou em vigor em março de 2012, e a partir deste momento todos os alunos passaram a cursá-lo. Para isto foi construído um quadro de equivalências, que teve vigência até o segundo semestre de 2016, período no qual foi iniciada avaliação da percepção dos discentes e docentes quanto aos resultados do mesmo.

\section{TENSÕES DO PROCESSO E CONSIDE- RAÇÕES FINAIS}

Os principais desafios durante o processo de maturação de um PPC da área da saúde foram descritos por Salvador; Sant'Ana $(2017)^{11}$. O primeiro seria a necessidade de investimento na formação dos docentes para prepará-los para este desafio; o segundo seria aproximar a instituição das gestões dos serviços públicos de saúde, visando fortalecer as parcerias para que as atividades que envolvam ensino-serviço aconteçam de forma conveniente; o terceiro desafio seria tornar o projeto pedagógico a principal ferramenta para mudanças de gestão e qualidade do ensino, por meio de sua avaliação contínua, e para isto a contribuição de todos os atores é necessária.
No primeiro semestre de implantação do currículo da Faculdade de Odontologia, os principais problemas foram relacionados com os recursos humanos envolvidos no processo. Os discentes, que estavam acostumados a ter aulas em ambulatórios específicos, nos quais somente realizavam procedimentos de uma especialidade, se depararam com disciplinas de Clínicas Integradas por perfis de complexidade e tiveram como primeira impressão que realizariam menos procedimentos específicos em comparação ao currículo antigo.

Era comum escutar dos discentes nos corredores frases como estas: “...antes fazíamos seis tratamentos endodônticos na disciplina de Endodontia, agora só realizamos dois tratamentos na disciplina de Clínica Integrada III...". Para amenizar esta percepção errônea, a coordenação de curso realizava, regularmente, ciclos de reuniões com os representantes de sala, visando escutar a comunidade discente e esclarecer suas dúvidas para deixar claro que no currículo novo, por exemplo, ele faria os tratamentos endodônticos em várias disciplinas ao longo do curso e não mais em uma única disciplina concentrada em um semestre conforme o currículo antecessor.

O Corpo Docente estava em sua maioria empenhado para que o projeto pedagógico funcionasse a contento, porém também foram identificados núcleos de resistência alicerçados pelo tradicionalismo e pela insegurança do novo momento pelo qual todos estavam passando. $\mathrm{O}$ principal desafio estava na resistência de trabalho em grupo para fomentar a interdisciplinaridade e na avaliação integrada, uma vez que isso demanda tempo e muito mais esforço dos docentes quando comparado com a realidade anterior ${ }^{5}$.

Para estreitar o convívio entre docentes e discentes e assim favorecer a implementação do projeto pedagógico e a superação das dificuldades, o NDE e as instâncias da gestão da Faculdade realizaram em 4 semestres (2012 e 2013) o 
acompanhamento da execução da última versão do projeto pedagógico por meio de várias consultas formais aos docentes e aos discentes do curso.

As sugestões foram debatidas com os discentes em sala de aula e pelos docentes em duas semanas pedagógicas. Muitas das situações ou sugestões da comunidade acadêmica foram otimizadas por meio da realização de oficinas pedagógicas sobre plano de ensino integrado, trabalho em equipe, métodos de avaliação integrados e metodologias ativas de ensino.

Para facilitar o processo de trabalho, por sugestão do NDE e aprovação dos docentes, foi estabelecida, durante dois semestres letivos, uma carga-horária pedagógica quinzenal, na qual todos os professores foram alocados para reunião com suas equipes e realizar as atividades pertinentes ao planejamento, execução e acompanhamento de suas disciplinas. Estas atividades incluíam montagem dos planos de ensino em equipe, formulação de avaliações integradas, treinamento de metodologias ativas de ensino, dentre outras atividades pedagógicas que visem o bom funcionamento do PPC como um todo.

Este horário de alocação docente em comum também foi utilizado para realização de reuniões, treinamentos ou oficinas, pois não havia aula de graduação neste horário durante todo semestre letivo, oferecendo, assim, oportunidade para que todos os professores pudessem se reunir ao mesmo tempo para discussões.

O Colegiado de Curso se empenhou em analisar semestralmente os planos de ensino, dando sugestões para que todas as disciplinas realizassem pelo menos uma metodologia ativa e que os métodos avaliativos estivessem bem descritos e contemplassem a integralidade. A Coordenação Acadêmica se empenhou em realizar grupos de trabalho para discussão e treinamento de protocolos clínicos, como os de terapêutica medicamentosa, uso de fluoretos e diagnóstico e manejo de cárie. Estas discussões tinham o objetivo de calibrar todo corpo docente, uma vez que estariam atuando em ambulatórios integrados.

Em artigo publicado por Althaus (2017) ${ }^{14}$ fica clara a importância das metodologias ativas no contexto universitário, pois são baseadas na mediação do processo de ensino visando à construção do conhecimento colaborativo. Por se tratar de um ensino diferente quando comparado ao ensino tradicional bancário, é necessário o treinamento tanto dos docentes quanto dos discentes para que a metodologia ativa aconteça de modo horizontal e tenha bons resultados.

Estas atividades foram mantidas pela unidade nos anos de 2014 a 2016 onde outras tensões influenciaram o andamento das atividades, pois a infraestrutura da unidade e o número de docentes não foi otimizado na velocidade necessária para colhermos melhores frutos, atual foco de atenção pela gestão da faculdade.

Após sete anos da implementação do novo projeto pedagógico é possível compreender as análises relatadas por Hypolito $(2014)^{15}$, que versam sobre a articulação do projeto pedagógico com a gestão e a avaliação escolar. Um dos pilares desta articulação seria a construção coletiva do projeto pedagógico, de forma que todos os atores que vivem naquela realidade participem das mudanças e para isto o tempo de planejamento é fundamental. Esta construção deve dar motivação para que o ensino se torne melhor e para isto o projeto deve ser alicerçado na realidade cultural da escola e da comunidade e não só em diretrizes governamentais.

Outro ponto analisado por Hypolito (2014) ${ }^{15}$ é a tendência das escolas balizarem seu planejamento escolar somente pelas DCN ou pelos exames nacionais de avaliação que geram uma distorção na formação dos alunos e não garantem qualidade de melhoria na escola. No ensino superior brasileiro, o principal índice de avaliação é resultado do Exame Nacional de Desempenho de Estudantes (Enade), parte integrante do Sistema 
Nacional de Avaliação da Educação Superior (Sinaes). Nesta avaliação, a Faculdade de Odontologia da UFAM permaneceu durante 4 edições com nota 4 e em 2016 obteve nota 5, resultado este que injetou motivação para a comunidade continuar caminhando para atingir um objetivo em comum, a melhora contínua da qualidade do curso e da convivência entre seus atores.

\section{ABSTRACT \\ Curriculum redesign of the Dental school of the Federal University of Amazonas}

The aim of the paper is to report the experience of faculty members of a Dental school during the process of developing and implementing a new academic program (AP). The AP has been redesigned taking into consideration the characterization of the course by discussing the profile of alumni, physical structure, objectives, curriculum structure, academic internships and methodological design. The new curriculum was implemented in 2012 and it focuses on interdisciplinarity, integrated outpatient clinics and a mandatory traineeship that consists of more than $40 \%$ of the curriculum between intra- and extra-mural activities. Difficulties were encountered during the implementation of the new program and the academic community sought tools to minimize them. The new education program is dynamic, and it has been improving every year.

Descriptors: Education. Dentistry. Curriculum.

\section{AGRADECIMENTOS}

Aos professores da Faculdade de Odontologia, Gustavo Henrique Diniz Pimentel, Simone Assayag Hanan e Juliana Vianna Pereira; do Instituto de Ciências Biológicas, Maria Isabel Portus e da Faculdade de Educação, Lucíola Inês Pessoa Cavalcante e Michelle de Freitas Bissoli, da Universidade Federal do Amazonas pela relevante participação na construção do projeto pedagógico.

\section{REFERÊNCIAS}

1. Brito RM. 100 anos UFAM. 2nd ed. Manaus: EDUA; 2011.

2. Lemos CLS. A implantação das Diretrizes Curriculares dos Cursos de Graduação em Odontologia no Brasil: algumas reflexões. Rev ABENO. 2005;5(1):80-5.

3. Toassi RFC, Stobäus CD, Mosquera JJM, Moysés SJ. Currículo integrado no ensino de Odontologia: novos sentidos para a formação na área da saúde. Interface Comunic Saude Educ. 2012;16(41):52942.

4. Fadel CB, Baldani MH. Percepções de formandos do curso de odontologia sobre as diretrizes curriculares nacionais. Trab Educ Saúde. 2013;11(2):339-54.

5. Souza MCA, Casotti E, Mello ACF, Goyata FR, Souza TC, Albuquerque CJM. Interdisciplinaridade no Ensino Superior: de imagem-objetivo à realidade. Rev Bras Educ Méd. 2012;36(1 Suppl 2):158-63.

6. Werneck MAF, Senna MIBS, Drumond MM, Lucas SD. Nem tudo é estágio: contribuições para o debate. Ciênc Saúde Colet. 2010;15(1):221-31.

7. Oliveira ER, Codato LAB, Massaoka ST, Gabriel M. Programa de Educação pelo Trabalho para a Saúde: formação baseadaiches pressupostos das Diretrizes Curriculares Nacionais. Rev ABENO. 2011;11(1):43-6.

8. Santa Rosa TTA, Vargas AMD, Ferreira EF. O internato rural e a formação de estudantes do Curso de Odontologia da UFMG. Interface Comunic Saúde Educ. 2007;11(23):451-66.

9. Francisco KMS, Moimaz SAS, Diniz DG, Saliba NA. Oferta e Distribuição de 
Estágio Supervisionado em Cursos de Odontologia do Estado de Minas Gerais, Brasil. Biosci J. 2010;26(1):152-60.

10. Batista MJ, Gibilini C, Kobayashi HM, Fereira LL, Gonçalo CS, Sousa MLR. Relato de Experiência da interação entre universidade, comunidade e Unidade de Saúde da Família em Piracicaba, SP, Brasil. Arq Odontol. 2010;46(3):144-51.

11. Salvador LDS, Sant'ana PA. Projetos pedagógicos de cursos de saúde e sua articulação com as políticas públicas para a formação em saúde no Brasil. Rev Docência Ens Sup. 2017;7(1):185-202.

12. Cortela BSC. Práticas inovadoras no ensino de graduação na perspectiva de professors universitários. Rev Docência Ens Sup. 2016 Out; 6(2):9-34.

13. Teófilo TJS, Santos NLP, Baduy RS. Betting on change in medical education: trajectories of a medical school. Interface Comunic Saúde Educ. 2017;21(60):17788.
14. Althaus MTM, Bagio VA. As metodologias ativas e as aproximações entre o ensino e a aprendizagem na prática pedagógica universitária. Rev Docência Ens Sup. 2017;7(2):79-96.

15. Hypolito AM. Currículo e projeto políticopedagógico: implicações na gestão e no trabalho docente. Cad Educ. 2014; XVIII:11-26.

\section{Correspondência para:}

Emílio Carlos Sponchiado Júnior e-mail: spemilio@ufam.edu.br Universidade Federal do Amazonas Rua Waldemar Pedrosa, 1539 Praça 14 de Janeiro 69025-050 - Manaus/AM 